\title{
Massive haemoptysis in pregnancy
}

\author{
S Mothilal, MB ChB, FCP, MMed; A Esmail, MD, FCP, Cert Pulm; K Dheda, MB ChB, FCP, FRCP, PhD \\ Division of Pulmonology, Department of Medicine, University of Cape Town Lung Institute and Groote Schuur Hospital, Cape Town, South Africa
}

Corresponding author: S Mothilal (sh1kar@yahoo.com)

Haemoptysis in pregnancy may be due to a myriad of causes, including pulmonary embolism, heart failure, vasculitides and infective or post-infective aetiologies. Pulmonary arteriovenous malformations (PAVMs) are a rare cause of massive haemoptysis that involve an abnormal communication between the pulmonary arteries and the pulmonary veins. The majority of PAVMs are congenital and associated with hereditary haemorrhagic telangiectasia (HHT). To illustrate the complexity of the diagnosis and management of PAVM in pregnancy, we report the case of a 32 -year-old woman presenting with massive haemoptysis at 36 weeks of gestation.

S Afr Respir J 2016;22(4):101-103. DOI:10.7196/SARJ.2016.v22i4.104

\section{Case presentation}

A 32-year-old woman presented with a 1-day history of $\sim 500 \mathrm{~mL}$ of haemoptysis at 36 weeks' gestation. This was her first episode of haemoptysis, which was preceded by a dry cough for 6 months. She did not experience chest pain, orthopnoea, paroxysmal nocturnal dyspnoea or constitutional symptoms. Her two previous pregnancies were uncomplicated. She tested negative for HIV during antenatal screening and she had no comorbidities. She was not using any chronic medication or illicit drugs. She had a 10 pack-year smoking history and consumed alcohol in moderation prior to her pregnancy. She had no significant family history, travel history or environmental exposure.

On examination, her blood pressure was $130 / 80 \mathrm{mmHg}$, heart rate 90 beats per minute, respiratory rate 24 breaths per minute and temperature $36^{\circ} \mathrm{C}$. Her pulse oximetry measured $97 \%$ on ambient air. She exhibited digital clubbing but had no features to suggest deep vein thrombosis, petechia, purpura, telangiectasia or connective tissue disease. Her respiratory examination revealed a patent airway with bilateral equal breath sounds and no added sounds. Her heart sounds were normal with no murmurs. Her abdomen was soft and non-tender. Her neurological examination was normal.

She had a normal full blood count, renal function, liver function, coagulation studies, vasculitic screen and urinalysis. Her sputum microscopy, culture and mycobacterium tuberculosis polymerase chain reaction (GeneXpert MTB/RIF PCR) were negative. Her chest X-ray and computed tomography pulmonary angiogram (CTPA) with 3D reconstruction are shown in Figs 1, 2 and 3 , respectively.

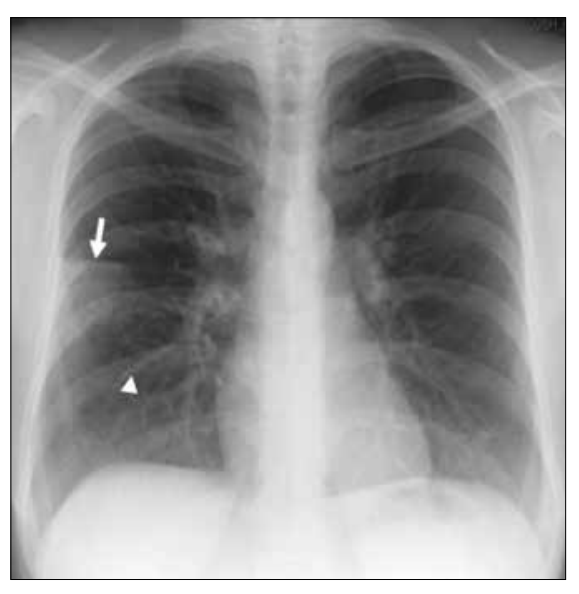

Fig. 1. Chest X-ray demonstrating a peripheral wedge-shaped opacity (arrow) in the right mid-zone and a linear opacity extending from the right hilum representing a feeding vessel (arrowhead). 
In view of the severity of the patient's symptoms and her abnormal chest X-ray, CTPA was regarded as the imaging modality of choice. The CTPA demonstrated a large pulmonary arteriovenous malformation (PAVM). The patient subsequently underwent successful coiling of her PAVM (Fig. 4) and had an uncomplicated vaginal delivery. Repeat CTPA confirmed retraction of the aneurysmal sac. Prior to discharge, the patient was counselled regarding the risks related to PAVM in future pregnancy and was offered contraception.

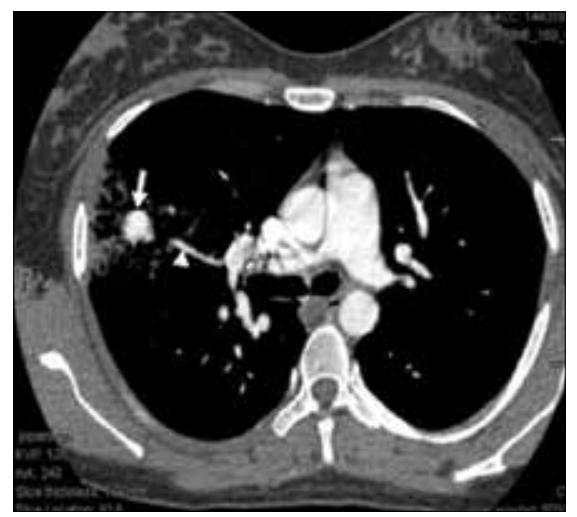

Fig. 2. Axial slice of CTPA demonstrating the pulmonary arteriovenous malformation (arrow) with surrounding haemorrhage and a feeding vessel (arrowhead) extending from the right hilum.

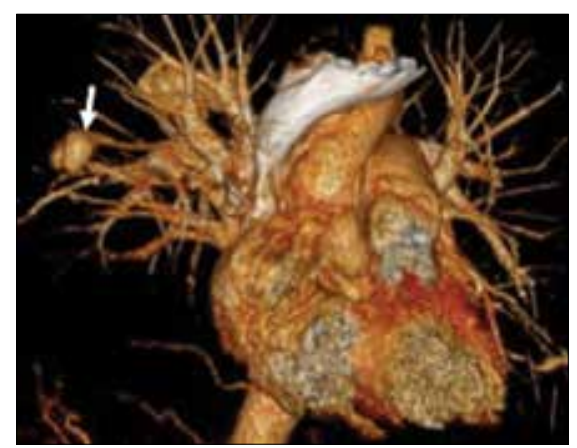

Fig. 3. $3 D$ reconstruction demonstrating the angio-architecture of the pulmonary circulation with the arrow showing the PAVM.

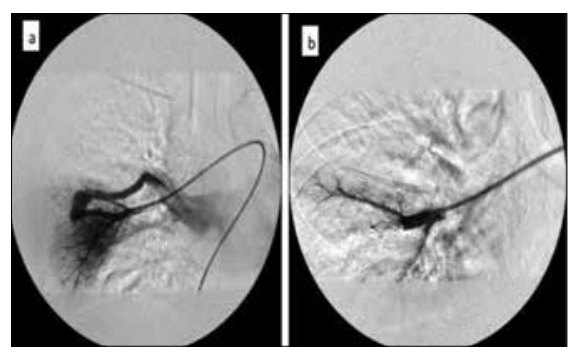

Fig. 4. Catheterisation (a) and successful coiling (b) of the PAVM.

\section{Discussion}

Haemoptysis in pregnancy may be due to a myriad of causes, including pulmonary embolism, heart failure, vasculitides and infective or post-infective aetiologies. Although no standardised definition exists, massive haemoptysis is generally associated with $\sim 500 \mathrm{~mL}$ of expectorated blood in a 24 hour period, or a rate of haemoptysis greater than $200 \mathrm{~mL}$ per hour. ${ }^{[1]}$ PAVMs are a rare cause of massive haemoptysis but should be considered in the differential diagnosis of a patient presenting with this clinical problem.

PAVMs were first described in 1897. ${ }^{[2]}$ They consist of abnormal communications between the pulmonary arteries and the pulmonary veins. Ninety-five percent of the feeding arteries arise from the pulmonary circulation, while $5 \%$ arise from the bronchial artery, internal mammary artery or descending aorta. Furthermore, $53-70 \%$ of PAVMs are found in the lower lobes and are commonly multiple. ${ }^{[3]}$

Over $80 \%$ of PAVMs are congenital, and of these, $47-96 \%$ (depending on the context) are associated with the autosomal dominant disorder hereditary haemorrhagic telangiectasia (HHT), also known as OslerWeber-Rendu disease. Conversely, it is

\section{Table 1. Aetiology of PAVM}

Primary

HHT

Secondary

Congenital

Chest trauma

Thoracic surgery

Hepatic cirrhosis

Metastatic carcinoma

Mitral stenosis

Infections (actinomycosis, schistosomiasis)

Systemic amyloidosis estimated that, overall, 5 - 15\% with HHT have a PAVM. ${ }^{[4]}$ Secondary or acquired causes of PAVM, although uncommon, have also been reported (Table 1).

The pathogenesis of PAVMs is poorly understood. ${ }^{[4]}$ It is proposed that a defect in the terminal arteriole occurs, leading to dilatation of thin-walled capillary sacs. Blood then bypasses the normal oxygen-exchanging pulmonary capillary bed, returning to the pulmonary veins in a desaturated state. The fundamental defect is therefore a right-to-left shunt. ${ }^{[5]}$

Dyspnoea, especially with exercise, develops over many years. ${ }^{[6]}$ In severe cases, dyspnoea in the upright position (platypnoea) with associated cyanosis (orthodeoxia) is present. Haemoptysis occurs rarely, accounting for $0.2 \%$ of all causes of haemoptysis, ${ }^{[7]}$ which may sometimes be massive. On examination, patients may demonstrate digital clubbing while the presence of telangiectasia is a valuable clue to the association with HHT. Machinery murmurs or bruits are heard over large PAVMs. Hypoxia, if present, does not correct fully after the administration of $100 \%$ oxygen. PAVMs are also associated with a variety of complications, some of which are life-threatening, including strokes, brain abscesses and heart failure.

Chest X-ray is an important diagnostic tool and shows abnormalities in more than $90 \%$ of patients. ${ }^{[8]}$ CTPA, however, remains the gold standard for evaluating the angio-architecture of the PAVM, and when combined with 3D helical scanning can produce images of the entire vascular structure with a sensitivity of up to $95 \% .{ }^{[9]}$ The classical radiographical features of PAVM are a rounded, sharply defined mass of uniform density, frequently lobulated, and ranging from $1-5 \mathrm{~cm}$ in diameter. There may sometimes be a connecting vessel radiating from the hilum.

Until 1977, surgery was the only method of treatment, in which ligation, local excision,

Table 2. Indications and complications of embolotherapy

\begin{tabular}{ll}
\hline Indications & Complications \\
\hline Hypoxaemia & Device migration \\
Haemoptysis & Haemorrhage \\
Paradoxical embolisation & Vascular injury \\
Feeding vessels $2-3 \mathrm{~mm}$ & Arterial/venous thrombosis \\
Progressive enlargement &
\end{tabular}


lobectomy or pneumonectomy was performed. Nowadays, embolotherapy is the treatment of choice as it avoids major surgery, anaesthesia, loss of lung parenchyma and additional cost. ${ }^{[10]}$ Moreover, embolotherapy has comparable success rates with surgery. ${ }^{[11]}$ It is performed by catheterisation of the feeding artery and either coiling or balloon deployment into the PAVM. The indications and complications of embolotherapy are listed in Table 2.

Untreated, PAVMs confer a high mortality risk of up to $\sim 30 \%{ }^{[12]}$ Furthermore, genetic counselling is an important aspect of management in patients with HHT. ${ }^{[13]}$

\section{PAVM in pregnancy}

PAVM in pregnancy is rare and is associated with an increased rate of growth and complications such as haemoptysis. ${ }^{[14]}$ This is due to the increase in blood volume and the vasodilatory effect of pregnancy hormones, most marked during the second trimester, which leads to increased pulmonary blood flow, PAVM dilatation and subsequent rupture. Therefore, female patients with a history of PAVM must be counselled regarding the substantial risks associated with pregnancy and be offered suitable contraception.

\section{Conclusion}

PAVM is a rare cause of massive haemoptysis, however it must be considered in the differential diagnosis of a patient presenting with this clinical problem. Embolotherapy is the treatment of choice for symptomatic or large lesions. Furthermore, PAVMs are associated with an increased rate of complications in pregnancy, and female patients should be counselled regarding this risk and offered contraception.

1. Jean-Baptiste E. Clinical assessment and management of massive hemoptysis. Crit Care Med 2000;28(5):1642-1647. http://dx.doi.org/10.1097/00003246-200005000-00066

2. Churton T. Multiple aneurysms of the pulmonary artery. Br Med J 1897;1:1223-1225.

3. Boscher Lea. An analysis of the pathologic anatomy of pulmonary arteriovenous aneurysms with particular reference to the applicability of local excision. Surgery 1959;45:91-104.

4. Hodgson $\mathrm{CH}$. Hereditary hemorrhagic telangiectasis and pulmonary arteriovenous fistula. N Engl J Med 1959;26(3)1:625-636. http://dx.doi.org/10.1056/ nejm195909242611301

5. Gossage JR, Kanj G. Pulmonary arteriovenous malformations. A state of the art review. Am J Respir Crit Care Med 1998;158(2):643-661. http://dx.doi. org/10.1164/ajrccm.158.2.9711041

6. Moyer JH, Glantz G, Brest A. Pulmonary arteriovenous fistulas: Physiologic and clinical considerations. Am J Med 1962;32(3):417-425. http:// dx.doi.org/10.1016/0002-9343(62)90131-6
7. Abdulmalak C, Cottenet J, Beltramo G, et al. Haemoptysis in adults: A 5-year study using the French nationwide hospital administrative database. Eur Respir J 2015;46(2):503-511. http://dx.doi. org/10.1183/09031936.00218214

8. Dines DE, Arms RA, Bernatz PE, Gomes MR Pulmonary arteriovenous fistulas. Mayo Clin Proc 1974;49:460-465. http://dx.doi.org/10.1016/s00034975(10)66398-9

9. Remy J, Remy-Jardin M, Wattinne L, Deffontaines C. Pulmonary arteriovenous malformations: Evaluation with CT of the chest before and after treatment. Radiology 1992;182(3):809-816. http://dx.doi. org/10.1148/radiology.182.3.1535899

10. Meek ME, Meek JC, Beheshti MV. Management of pulmonary arteriovenous malformations. Semin Intervent Radiol 2011;28(1):24-31. http://dx.doi. org/10.1055/s-0031-1273937

11. Saluja S, Sitko I, Lee DW, Pollak J, White RI Jr Embolotherapy of pulmonary arteriovenous malformations with detachable balloons: Longterm durability and efficacy. J Vasc Interv Radiol 1999;10(7):883-889. http://dx.doi.org/10.1016/s1051 0443(99)70132-6

12. Khurshid I, Downie GH. Pulmonary arteriovenous malformation. Postgrad Med J 2002;78(918):191-197. http://dx.doi.org/10.1136/pmj.78.918.191

13. Guttmacher AE, Marchuk DA, White RI Jr. Hereditary hemorrhagic telangiectasia. N Engl J Med 1995;333(14):918-924. http://dx.doi.org/10.1056/ nejm199510053331407

14. Ference BA, Shannon TM, White RI, Jr, Zawin M, Burdge CM. Life-threatening pulmonary hemorrhage with pulmonary arteriovenous malformations and hereditary hemorrhagic telangiectasia. Chest 1994;106(5):1387-1390. http://dx.doi.org/10.1378/ chest.106.5.1387 